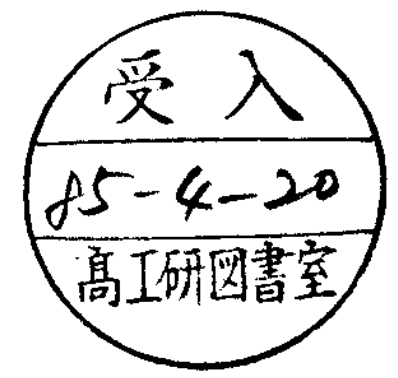

\section{GAUGINO MASSES AND GRAND UNIFICATION}

John Ellis, K. Enquist, D.V. Nanopoulos

CERN - Geneva

and

K. Tamvakis

Max-Planck-Institut für Physik und Astrophysik Werner-Helsenberg-Institut fuir Physik Munich, FRG and

Physics Department, University of Ioannina Ioannina, Greece

\section{ABSTRACT}

Neither the $\mathrm{SU}(3), \mathrm{SU}(2)$ and $\mathrm{U}(1)$ gauge couplings nor the gaugino masses need be universal at the grand unification scale $M_{X}$ in supergravity theories. The experimental value of $\sin ^{2} \theta_{W}$ is naturally reproduced only if $M_{X}$ is somewhat less than the Planck Mass $M_{P}$. In this case with $\mathrm{SU}(5)$ broken by an adjoint 24 of Hifgs there is a simple sum rule relating the $\mathrm{SU}(3), \mathrm{SU}(2)$ and $\mathrm{U}(1)$ gaugino masses $n_{1}$. Requiring that the supersymmetry breaking lepto-quark mass splitting vanish as in noscale models would impose the specific nonuniversal ratios $m_{3}: m_{2}: m_{1}=5:-5:-1$ at $\mathrm{M}_{\mathrm{X}}$. In this case $m_{\tilde{y}} / \mathrm{m}_{\mathrm{g}}$ is half the usual value derived from universality, while $\mathrm{m}_{\mathrm{b}} / \mathrm{m}_{\tau}$ takes its usual value. 
With the recent discovery ${ }^{1)}$ of the intermediate gauge bosons at the CERN ppcollider, one of the central predictions of the $\mathrm{SU}(3) \times \mathrm{SU}(2) \times \mathrm{U}(1)$ standard model has been confirmed. Although the standard model is not completely tested, there is a growing belief among physicists that it describes physics correctly at least up to energies of the order of the electroweak scale. A theoretically unsatisfying feature of the model however, is that it contains a number of free parameters whose determination requires one to go beyond $\mathrm{SU}(3) \times \mathrm{SU}(2) \times \mathrm{U}(1)$. This has led to the enterprise of grand unification in which the standard gauge group is unified in a larger group such as $\mathrm{SU}(5)$ at an exponentially distant mass scale. Parameters such as the electroweak mixing angle or quark to lepton mass ratios, which were free in the framework of $S U(3) \times S U(2) \times U(1)$, can now be determined in remarkable agreement with measured values. Supersymmetry has been introduced in the unification programme as an improvement over conventional perturbatively renormalizable gauge field theories since it automatically solves the technical aspects of the gauge hierarchy problem associated with fundamental scalars ${ }^{2}$. More specifically, unification in the framework of $N=1$ local supersymmetry (supergravity) offers unexpected fruits such as a possible understanding of the origin of the electroweak scale itself and thus completely solving the gauge hierarchy problem of conventional GUTs, as well as Improving enormously our understanding of the absence of any observable cosmological constant ${ }^{3)}$. Unification in the framework of $N=1$ supergravity should of course reproduce the standard predictions of $\sin ^{2} \theta_{W}$ and $m_{b} / m_{\tau}$ as well as a set of relations among the parameters associated with the new particles of the theory. In particular, one would naturally expect to have mass relations among gauge fermions.

In this paper, under general assumptions we derive and analyze the gauge fermion mass relations implied by unification. Contrary to the chiral sector of the theory whose spectrum is determined in a mode1-dependent way, gauge fermion masses are strong1y constrained by the unifying group and at the same time related in a model-independent way.

Let us consider a supersymmetric $\mathrm{SU}(5)$ gauge theory with an arbitrary chiral matter superfield content coupled to $N=1$ supergravity. All gauge and matter terms in the component Lagrangian are expressible in terms of two fundamental functions of chiral superfields ${ }^{4)}$. These are a chiral function $f_{\alpha \beta}(\phi)$ which transforms as the symmetric product of two adjoint representations [ In the case of $\operatorname{SU}(5) \alpha, \beta=1, \ldots, 24]$ and is an analytic function of the left-handed chiral superfields $\phi_{i}$, and the Kähler potential $G\left(\phi_{i}, \phi_{i}^{*}\right)$ which is a singlet under the gauge group and is a real function of chiral superfields. The structure of the 
kinetic terms of gauge superfields is determined by $f_{\alpha \beta}$. The same term in the superspace Lagranglan that gives rise to gauge supermultiplet kinetic terms also generates bilinear gauge fermion terms that lead to gaugino masses when supersymmetry is broken. In component field form the Lagrangian reads (we use natural units in which $M_{\mathrm{P}} / \sqrt{8 \pi}=1$ )

$$
\begin{aligned}
& e^{-1} \mathcal{L}=\frac{1}{2} \operatorname{Re} f_{\alpha \beta}\left(-\frac{1}{2} \bar{\lambda}^{\alpha} \not \partial \lambda^{\beta}\right)-\frac{i}{8} \operatorname{Im} f_{\alpha \beta} e^{-1} D_{\mu}\left(e^{\bar{\lambda}^{\alpha}} \gamma^{\mu} \gamma_{5} \lambda^{\beta}\right) \\
& -\frac{1}{4} \operatorname{Re} f_{\alpha \beta} F_{\mu \nu}^{\alpha} F^{\beta \mu \nu}+\frac{i}{4} \operatorname{Im} f_{\alpha \beta} F_{\mu \nu}^{\alpha} \tilde{F}^{\beta \mu \nu} \\
& +\frac{1}{4} e^{-G / 2} G^{i}\left(G^{-1}\right)_{i}^{j} \frac{\partial f_{\alpha \beta}^{*}}{\partial \phi^{* j}} \lambda^{\alpha} \lambda^{\beta}+\text { h.c. }+\cdots
\end{aligned}
$$

in the notation of Cremmer et al. ${ }^{4)}: G^{i} \equiv \partial G / \partial \phi_{1}$ and $\left(G^{-1}\right)_{1}^{j}$ is the inverse matrix of $G_{i}^{j} \equiv \partial G / \partial \phi *^{i} \partial \phi_{j}$.

In the minimal case $\left(f_{\alpha \beta}=\delta_{\alpha \beta}\right)$, the gauge kinetic terms acquire their familiar form and the gaugino mass term vanishes. A non-trivial $f_{\alpha \beta}$ will in principle be a function of all chiral superfields, but we need only consider those that get large expectation values. Such are gauge-singlet fields $z_{1}$ that make up the "hidden" sector, and "observable" flelds associated with the SU(5) breakdown to $\mathrm{SU}(3) \times \mathrm{SU}(2) \times \mathrm{U}(1)$, such as a chiral superfield in the adjoint (24) representation $\Sigma_{j}^{i}=\Sigma^{\alpha}\left(\lambda_{\alpha}\right)_{j}^{i} / \sqrt{2}$. The SU(5) breaking can also be achieved with a field in the 75 representation ${ }^{5)}$ but for the moment let us restrict ourselves to the simplest case of the 24 and come back later to the 75 . Keeping in mind that $f_{\alpha \beta}$ transforms as the symmetric product of two adjoint representations, the most general form it could have is

$$
f_{\alpha \beta}=A(z, \ldots) \delta_{\alpha \beta}+B(z, \ldots) d_{\alpha \beta \gamma} \Sigma^{\gamma}
$$

plus higher order terms in $\left.\Sigma 11 k e \mathrm{c}(\mathrm{z}, \ldots) \Sigma_{\alpha} \Sigma_{\beta}{ }^{\star}\right)$, etc. As we will see later, to explain in a natural way the nearness of $\sin ^{2} \theta_{W}$ to conventional GUT predictions, we will need to assume $\langle 0|\Sigma| 0\rangle\langle<1$, in which case higher orders may be neglected. In Eq. (2), A and B are

*) Such terms give contributions to both $f_{\alpha \beta}$ and its derivatives suppressed by extra powers of $\Sigma$. We shall see later that 1 t is reasonable to ignore them. 
gauge invariant functions of the chiral superfields. Therefore, if we ignore all other fields except $z$ and $\Sigma$ for the reasons stated, they should be analytic functions of $z, \operatorname{Tr}\left(\Sigma^{2}\right)=\Sigma^{\alpha} \Sigma^{\alpha}$ and $d_{\alpha \beta \gamma} \Sigma^{\alpha} \Sigma^{\beta} \Sigma^{\gamma}$, although the latter two are negligible if $\langle 0|\Sigma| 0\rangle\left\langle\left\langle 1\right.\right.$, as we suppose. The group-theoretic symmetric coefficient $d_{\alpha \beta \gamma}$ is defined as $d_{\alpha \beta \gamma}=2 \operatorname{Tr}\left(\left\{\lambda_{\alpha} / 2, \lambda_{\beta} / 2\right\} \lambda_{\gamma} / 2\right)$ with $\lambda_{\alpha} / 2$ the generators of $\operatorname{SU}(5)$ in the adjoint representation.

Returning to the Lagrangian (1), we see that in order to regain the familiar minimal form of the kinetic terms

$$
\frac{1}{2}\left(-\frac{1}{2} \bar{\lambda}^{\alpha} \not \partial \hat{\lambda}^{\alpha}\right)-\frac{1}{4} \hat{F}_{\mu \nu}^{\alpha} \hat{F}^{\alpha \mu \nu}
$$

the gauge flelds have to be rescaled according to

$$
F_{\mu \nu}^{\alpha}\left(f^{\alpha}\right)^{\frac{1}{2}}=\hat{F}_{\mu \nu}^{\alpha} ; A_{\mu}^{\alpha}\left(f^{\alpha}\right)^{\frac{1}{2}}=\hat{A}_{\mu}^{\alpha} ; \lambda^{\alpha}\left(f^{\alpha}\right)^{\frac{1}{2}}=\hat{\lambda}^{\alpha}
$$

where the coefficients $f_{\alpha}$ are taken from

$$
\begin{aligned}
f_{\alpha \beta}\left(z_{0}, \Sigma_{0}\right) & =f_{\alpha} \delta_{\alpha \beta}=A\left(z_{0}, \ldots\right) \delta_{\alpha \beta}+d_{\alpha \beta \gamma} \Sigma_{0}^{\gamma} B\left(z_{0}, \ldots\right) \\
& =\left[A\left(z_{0}, \ldots\right)+\Sigma_{0} B\left(z_{0}, \ldots\right) C_{\alpha}\right] \delta_{\alpha \beta}
\end{aligned}
$$

with $z_{0}$ and $\Sigma_{0}^{\gamma}=\delta^{\gamma, 24} \Sigma_{0}$ the values of the scalar fields at the minimum. The representation invariants $d_{\alpha \beta 24} \equiv c_{\alpha} \delta \beta$ are

$$
C_{\alpha}= \begin{cases}2 / \sqrt{15} & (\alpha=1, \ldots, 8) \\ -1 / 2 \sqrt{15} & (\alpha=9, \ldots, 20) \\ -3 / \sqrt{15} & (\alpha=21,22,23) \\ -1 / \sqrt{15} & (\alpha=24)\end{cases}
$$

where we have assumed for simplicity that $f_{\alpha \beta}$ is real, but our results are true more generally. The rescaling conditions (3) go together with the definition of rescaled gauge couplings $g_{\alpha}$ according to

$$
g_{\alpha} f_{\alpha}^{1 / 2}=g
$$

Thus, the first striking consequence of a non-trivial $f_{\alpha \beta}$ is the modification of the standard unification condition at the unification scale ${ }^{M}{ }^{*}$ Now, this 
condition reads

$$
\alpha_{G}=\alpha_{3}\left(M_{X}\right) f_{3}=\alpha_{2}\left(M_{X}\right) f_{2}=\frac{5}{3} \alpha^{\prime}\left(M_{X}\right) f_{1}
$$

The gauge couplings are no longex equal at $M_{X}$. The classic renormalization group analysis that led to the predictions of $\sin ^{2} \theta_{W}$ and $M_{X}$ has to be re-examined and we intend to do so later.

Let us now analyze the gaugino mass matrix

$$
m_{\alpha \beta}=\frac{1}{4} e^{-G / 2} G^{i}\left(G^{-1}\right)_{i}^{j} \frac{\partial f_{\alpha \gamma}^{*}}{\partial \phi^{* j}} f_{\gamma \beta}^{-1}
$$

Using SU(5) covariance, the Kähler-potential-dependent factor can always be written as

$$
G^{i}\left(G^{-1}\right)_{i}^{j}=P_{1}\left(z_{0}, \Sigma_{0}\right) \delta^{j z}+P_{2}\left(z_{0}, \Sigma_{0}\right) \delta^{j \gamma} \Sigma_{0}^{* \gamma}
$$

(in no-scale models $\left.{ }^{3}\right) P_{2}=0$ ). Finally, we get

$$
m_{\alpha \beta}=\delta_{\alpha \beta} m_{3 / 2} \frac{\Gamma+c_{\alpha} \Delta \Sigma_{0}}{A+c_{\alpha} \Sigma_{0} B}
$$

where as a shorthand notation we have introduced

$$
\begin{aligned}
\Gamma & \equiv \frac{1}{4}\left\{P_{1}\left(\frac{\partial A}{\partial z}\right)_{0}+2 P_{2} \Sigma_{0}^{2}\left(\frac{\partial A}{\partial \Sigma^{2}}\right)_{0}\right\} \\
\Delta & =\frac{1}{4}\left\{P_{1}\left(\frac{\partial B}{\partial z}\right)_{0}+P_{2} B+2 \Sigma_{0}^{2}\left(\frac{\partial B}{\partial \Sigma^{2}}\right)_{0}\right\}
\end{aligned}
$$

Thus, grand unification in general allows different gaugino masses at $M$, contxary to the commonly assumed equal values. Equation (10) can be put in a more transparent form using Eqs. (4) and (6). Then, 
or

$$
m_{\alpha}=m_{3 / 2}\left\{\Gamma+c_{\alpha} \Delta \Sigma_{0}\right\} f_{\alpha}^{-1}=m_{3 / 2}\left(\frac{g_{\alpha}^{2}}{g^{2}}\right)\left\{\Gamma+c_{\alpha} \Delta \Sigma_{0}\right\}
$$

$$
\frac{m}{g_{\alpha}^{2}}=\frac{m_{3 / 2}}{g^{2}}\left\{\Gamma+c_{\alpha} \Delta \Sigma_{0}\right\} \quad\left(\text { at } M_{x}\right)
$$

It is already evident that since $m_{\alpha}$ depends only on two arbitrary functions $\Gamma$ and $\Delta$, we must find relations among the different gaugino masses. Substituting the group factor $c_{\alpha}$, we get

$$
\begin{aligned}
& \frac{m_{3}}{\alpha_{3}}=\frac{m_{3 / 2}}{\alpha_{G}}\left\{\Gamma+\frac{2 \Delta \Sigma_{0}}{\sqrt{15}}\right\} \\
& \frac{m_{2}}{\alpha_{2}}=\frac{m_{3 / 2}}{\alpha_{G}}\left\{\Gamma-\frac{3 \Sigma_{0} \Delta}{\sqrt{15}}\right\} \\
& \frac{m_{1}}{\frac{5}{3} \alpha^{\prime}}=\frac{m_{3 / 2}}{\alpha_{G}}\left\{\Gamma-\frac{\Sigma_{0} \Delta}{\sqrt{15}}\right\}
\end{aligned}
$$

which imply

$$
\frac{m_{3}}{\alpha_{3}}=-\frac{3}{2} \frac{m_{2}}{\alpha_{2}}+\frac{5}{2} \frac{m_{1}}{\frac{5}{3} \alpha^{\prime}}
$$

Since the ratios $m_{i} / \alpha_{i}$ are renormalization group invariant, the same relation holds for all energy scales $\mu<M_{X}$ * Relation (14) depends only on the assumption that $\mathrm{SU}(5)$ is broken to $\mathrm{SU}(3) \times \mathrm{SU}(2) \times U(1)$ with a non-vanishing expectation value of the adjoint $\Sigma$ which is < 1 . No assumption was made on the form of the Kähler potential (i.e., $P_{1}$ and $P_{2}$ ) or on the form of $A$ and $B$. If we were to include in $f_{\alpha \beta}$ a term of the form $c\left(z, \Sigma^{2}, \ldots\right) \Sigma_{\alpha} \Sigma_{\beta}$, the expression for $m_{1}$ would change to 
$-6-$

$$
\frac{m_{1}}{\frac{5}{3} \alpha^{\prime}}=\frac{m_{3} / 2}{\alpha_{G}}\left\{\Gamma-\frac{\Sigma_{0} \Delta}{\sqrt{15}}+\Sigma_{0}^{2} E\right\}
$$

where

$$
E \equiv P_{1} \frac{\partial C}{\partial z}+2 P_{2}\left(C+\Sigma_{0}^{2} \frac{\partial C}{\partial \Sigma^{2}}\right)
$$

This extra term, however, is suppressed even if $\Sigma_{0}$ is just an order of magnitude below $\mathrm{M}_{\mathrm{P}}$.

In the case that we set the heavy lepto-quark gaugino Majorana masses equal to zero, as required if their loops are not to make large contributions to the vacuum energy, which would be disastrous in many models ${ }^{6}$ ), we obtain an additional constraint. Indeed, for

$$
m_{\tilde{X}, \tilde{Y}}=m_{3 / 2} \frac{\Gamma-\Sigma_{0} \Delta / 2 \sqrt{15}}{A-\Sigma_{0} B / 2 \sqrt{15}}=0
$$

we get

$$
\begin{aligned}
& \frac{m_{3}}{\alpha_{3}}=5\left(\frac{m_{3 / 2} \Sigma_{0} \Delta}{2 \sqrt{15} \alpha_{G}}\right) \\
& \frac{m_{2}}{\alpha_{2}}=-5\left(\frac{m_{3 / 2} \Sigma_{0} \Delta}{2 \sqrt{15} \alpha_{G}}\right) \\
& \frac{m_{1}}{\frac{5}{3} \alpha^{1}}=-\left(\frac{m_{3 / 2} \Sigma_{0} \Delta}{2 \sqrt{15} \alpha_{G}}\right)
\end{aligned}
$$

Therefore, in this case the gaugino masses scale as 
$-7-$

$$
\frac{m_{3}}{\alpha_{3}}: \frac{m_{2}}{\alpha_{2}}: \frac{m_{1}}{\frac{5}{3} \alpha^{\prime}}=5:-5:-1
$$

This is in sharp contrast to the usually assumed equality $m_{3}\left(M_{X}\right)=m_{2}\left(M_{X}\right)=$ $m_{1}\left(M_{X}\right)$ which together with $\alpha_{3}\left(M_{X}\right)=\alpha_{2}\left(M_{X}\right)=(5 / 3) \alpha^{\prime}\left(M_{X}\right)=\alpha_{G}$ leads to

$$
\frac{m_{3}}{\alpha_{3}}=\frac{m_{2}}{\alpha_{2}}=\frac{m_{1}}{\frac{5}{3} \alpha^{1}}
$$

It should be pointed out also that if we neglect neutral Highs fermion mixings, as is appropriate for $m_{1,2} \ll M_{W}$, the photino now has a mass

$$
m \tilde{r}=\frac{4}{3} \sin ^{2} \theta_{w} m_{2}
$$

In contrast to the usual expression ${ }^{7}$ ) which is twice as large.

As we have already stated, our mass relation (14) can change if $S U(5)$ is broken down ${ }^{5}$ ) to $\mathrm{SU}(3) \times \mathrm{SU}(2) \times \mathrm{U}(1)$ with the help of the 75 representation whose expectation value is

$$
\sum_{j l}^{[i j]}=\Sigma_{0}\left\{\Delta_{c j}^{[i} \Delta_{c l}^{j]}+2 \Delta_{w j}^{[i} \Delta_{w l}^{k]}-\frac{1}{2} \delta_{j}^{[i} \delta_{l}^{k]}\right\}
$$

with

$$
\Delta_{C}=\operatorname{diag}(1,1,1,0,0) ; \Delta_{w}=\operatorname{diag}(0,0,0,1,1)
$$

The corresponding choice for $f_{\alpha \beta}$ is then

$$
\begin{aligned}
f^{\alpha \beta} & =A\left(z, \operatorname{Tr} \Sigma^{2}\right) \delta^{\alpha \beta}+B\left(z, \operatorname{Tr} \Sigma^{2}\right) \lambda_{[i}^{\alpha} \lambda_{k]}^{\beta l} \sum_{j l}^{[i k]} \\
& \equiv\left(A+B \Sigma_{0} D^{\alpha}\right) \delta^{\alpha \beta}
\end{aligned}
$$


The representation coefficients $D^{\alpha}$ are calculated to be

$$
D^{\alpha}=\left\{\begin{array}{cl}
-1 & (\alpha=1, \ldots, 8) \\
1 & (\alpha=9, \ldots, 20) \\
-3 & (\alpha=21,22,23) \\
5 & (\alpha=24)
\end{array}\right.
$$

Finally, the gaugino masses are

$$
\begin{aligned}
& \frac{m_{3}}{\alpha_{3}}=\frac{m_{3 / 2}}{\alpha_{a}}\left\{\Gamma^{\prime}-\Delta^{\prime}\right\} \\
& \frac{m_{2}}{\alpha_{2}}=\frac{m_{3} / 2}{\alpha_{G}}\left\{\Gamma^{\prime}-3 \Delta^{\prime}\right\} \\
& \frac{m_{1}}{\frac{5}{3} \alpha^{\prime}}=\frac{m_{3 / 2}}{\alpha_{G}}\left\{\Gamma^{\prime}+5 \Delta^{\prime}\right\}
\end{aligned}
$$

and the mass relation that follows is quite different from the one previously obtained

$$
\frac{m_{3}}{\alpha_{3}}=\frac{3}{4} \frac{m_{2}}{\alpha_{2}}+\frac{1}{4} \frac{m_{1}}{\frac{5}{3} \alpha^{\prime}}
$$

Again, in the case of vanishing lepto-quark gaugino Majorana masses we get

$$
\frac{m_{3}}{\alpha_{3}}: \frac{m_{2}}{\alpha_{2}}: \frac{m_{1}}{\frac{5}{3} \alpha^{\prime}}=-1:-2: 2
$$

which is also different from (16).

Let us now go back to the modified boundary condition for the gauge couplings $1 n$ the case with an adjoint 24 of Higgses $\Sigma$, and examine 1 ts consequences for the standard predictions of $\sin ^{2} \theta_{W}$ and $M_{X}$. The unification condition now reads

$$
\alpha_{3}\left(M_{X}\right)=\alpha_{2}\left(M_{x}\right) \frac{f_{2}}{f_{3}}=\frac{5}{3} \frac{f_{1}}{f_{3}} \alpha^{\prime}\left(M_{X}\right)=f_{3}^{-1} \alpha_{G}
$$

Notice that (23) requires $f_{3}, f_{2}, f_{1}>0$. Independently of any constraint, the f's satisfy an identity imposed by the fact that they a11 depend on only two 
$-9-$

arbitrary functions $A$ and $B$

$$
f_{3}=-\frac{3}{2} f_{2}+\frac{5}{2} f_{1}
$$

Since they must all be positive, we must in addition have

$$
f_{2}<\frac{5}{3} f_{1} \quad \text { or } f_{3}<\frac{5}{2} f_{1}
$$

The $f^{\prime} s$ do not appear in any expressions for the gaugino masses at any energy, since

$$
\frac{m_{\alpha}}{g_{\alpha}^{2}}=m_{3 / 2}\left\{\Gamma+c_{\alpha} \Sigma_{0} \Delta\right\} / g^{2}
$$

is a renormalization group invariant quantity. However, the renormalization group equations for the three gauge couplings certainly depend on the f's explicitly.

We find it convenient for later use to define

$$
m_{0} \equiv m_{3 / 2} \Gamma
$$

and

$$
\eta \equiv 5 / f_{3} \quad ; \xi \equiv 1 / f_{1}
$$

Then automatically $f_{2}=5 / 3(1 / \xi-2 / \eta)$, and the boundary condition at $M_{X}$ can be written

$$
\alpha_{G}=\frac{5 \alpha_{3}\left(M_{X}\right)}{\eta}=\frac{5}{3}\left(\frac{1}{\xi}-\frac{2}{\eta}\right) \alpha_{2}\left(M_{X}\right)=\frac{5 \alpha^{\prime}\left(M_{X}\right)}{3 \xi}
$$

$$
(\eta>2 \xi>0)
$$

The one-1oop renormalization group equations for the three gauge couplings are

$$
\begin{aligned}
& \alpha_{3}^{-1}(\mu)=\alpha_{3}^{-1}\left(M_{X}\right)-\frac{b_{3}}{2 \pi} \ln \frac{M}{\mu} x=\frac{5}{\eta \alpha_{G}}-\frac{b_{3}}{2 \pi} \ln \frac{M}{\mu} x \\
& \alpha^{-1}(\mu) \sin ^{2} \theta_{W}(\mu)=\alpha_{2}^{-1}\left(M_{X}\right)-\frac{b_{2}}{2 \pi} \ln \frac{M_{x}}{\mu}=\frac{5}{3 \alpha_{G}}\left(\frac{1}{3}-\frac{2}{\eta}\right)-\frac{b_{2}}{2 \pi} \ln \frac{M_{x}}{\mu} \\
& \frac{3}{5} \alpha^{-1}(\mu) \cos ^{2} \theta_{W}(\mu)=\frac{3}{5} \alpha^{\prime-1}\left(M_{X}\right)-\frac{b_{1}}{2 \pi} \ln \frac{M x}{\mu}=\frac{1}{3 \alpha_{G}}-\frac{b_{1}}{2 \pi} \ln \frac{M_{x}}{\mu}
\end{aligned}
$$


Defining

$$
D \equiv \frac{2 b_{3}}{3}\left(\frac{n}{3}-1\right)-\frac{5}{3} b_{1}-b_{2}
$$

we obtain

$$
\begin{aligned}
\ln \frac{M}{\mu} x= & \frac{2 \pi}{D}\left\{\alpha^{-1}(\mu)-\frac{2}{3}\left(\frac{\eta}{3}-1\right) \alpha_{3}^{-1}(\mu)\right\} \\
\sin ^{2} \theta_{W}(\mu)= & \frac{1}{3}\left(\frac{n}{3}-2\right) \alpha(\mu) \alpha_{3}^{-1}(\mu)+\left[1-\frac{2}{3} \alpha(\mu) \alpha_{3}^{-1}(\mu)\left(\frac{\mu}{3}-1\right)\right] \times \\
& \times\left[\frac{b_{3}}{3}\left(\frac{\eta}{3}-2\right)-b_{2}\right] D^{-1} \\
\alpha_{G}^{-1}=\frac{y}{5} & \left\{\alpha_{3}^{-1}(\mu)+b_{3}\left[\alpha^{-1}(\mu)-\frac{2}{3}\left(\frac{\eta}{3}-1\right) \alpha_{3}^{-1}(\mu)\right] D^{-1}\right\}
\end{aligned}
$$

In the minimal case of two Higgs doublets the renormalization group coefficients are $b_{3}=3, b_{2}=-1$ and $b_{1}=-33 / 5$.

If we now demand that the electroweak mixing angle lies in the experimentally observed ${ }^{8}$ ) range

$$
0.21 \leqslant \sin ^{2} \theta_{w}\left(M_{w}\right) \leqslant 0.23
$$

we find that the ratio $\eta / \xi$ is forced to take values in the rather narrow range $\left[\right.$ with $\left.\alpha_{3}\left(\mathrm{M}_{\mathrm{W}}\right)=0.122\right]$

$$
4.5 \lesssim \eta / \xi \lesssim 5
$$

This result holds independently of any possible vanishing of the supersymmetry breaking contribution to the masses of the $\widetilde{X}, \widetilde{Y}$ gauginos in the coset $\mathrm{SU}(5) / \mathrm{SU}(3) \times \mathrm{SU}(2) \times U(1)$. The behaviour of $\sin ^{2} \theta_{W}\left(M_{W}\right)$ as a function of $\eta / \xi 18$ shown in Fig. 1, where we also display the untfication mass $M_{X}$ as a function of $\eta / \xi$. As can be seen from there, the observed range of $\sin ^{2} \theta_{W}$ indicates that

$$
2.4 \times 10^{16} \mathrm{GeV} \leqslant M_{X} \leqslant 3.5 \times 10^{17} \mathrm{GeV}
$$

In Fig. 2 we show the behaviour of the combination $\eta \alpha_{G}$ as a function of $\eta / \xi$. Since in the experimentally favoured range (30) we find $M_{X}<M_{P}$, it presumably makes sense to demand that $\alpha_{G} \lesssim 1$, which implies that $\eta \gtrsim 0.2$. It is also inte- 
resting that in the range (30) the successful prediction for the ratio $m_{b} / m_{\tau}$ remains practically unchanged. In general we would obtain ${ }^{9}$ ) (at one-1oop)

$$
\frac{m_{b}}{m_{\tau}}=\left[\frac{5 \alpha_{3}(\mu)}{\eta \alpha_{G}}\right]^{\Gamma_{3}}\left[\frac{\alpha_{1}(\mu)}{\xi \alpha_{G}}\right]^{\Gamma_{1}}
$$

where $\Gamma_{i}=\left(\gamma_{\tau}^{(i)}-\gamma_{b}^{(i)}\right) / 2 b_{i}$, where $\gamma^{(i)}$ are the anomalous dimensions. With $\eta \alpha_{G} \sim 0.2$, the ratio (32) is practically equal to the "naive" SUSY value.

It is remarkable that the low-energy predictions (30)-(32) show such a consistency. In particular, the result (31) justifies a posteriori the neglect of terms of the type $\Sigma_{\alpha} \Sigma_{\beta}$ in $f_{\alpha \beta}$, which are suppressed by an extra power of $M_{X} / M_{P}$.

The spectrum of sparticles can now be expected to differ considerably from previous analyses ${ }^{10), 11)}$. If we parametrize the bare gaugino masses as

$$
m_{i}\left(M_{x}\right)=\xi_{i} m_{0} \quad(i=1,2,3)
$$

with $\underline{m}_{0}$ as defined in (26), the isodoublet squarks and right handed sleptons in radiative models will acquire masses

$$
\begin{aligned}
& m_{\tilde{q}}^{2}=m^{2}+c_{q}(\mu) m_{0}^{2} \\
& m_{\tilde{e_{R}}}^{2}=m^{2}+c_{R}(\mu) m_{0}^{2}
\end{aligned}
$$

where

$$
\begin{aligned}
& C_{q}(\mu)=\frac{16}{3} \xi_{3}^{2} J_{3}(\mu)+3 \xi_{2}^{2} J_{2}(\mu)+\frac{1}{15} \xi_{1}^{2} J_{1}(\mu) \\
& C_{R}(\mu)=\frac{12}{5} \xi_{1}^{2} J_{1}(\mu)
\end{aligned}
$$




$$
J_{i}(\mu) \equiv \frac{1}{2 b_{i}}\left[\left(\frac{\alpha_{i}(\mu)}{\alpha_{i}\left(M_{X}\right)}\right)^{2}-1\right]
$$

Setting $\sin ^{2} \theta_{W}\left(M_{W}\right) \simeq 0.21$ determines $\eta / \xi$ and $\alpha_{G} \eta$ (see Figs. 1 and 2 ). Let us now concentrate on the interesting case of vanishing supersymmetry breaking mass splitting in the lepto-quark vector supermultiplets as expressed by (16). At $\mu=$ $\mathrm{M}_{\mathrm{W}}$ we find that

$$
\begin{aligned}
& \frac{m_{\tilde{q}}^{2}}{m_{\tilde{g}}^{2}}=0.882+0.104\left(\frac{m}{\eta m_{0}}\right)^{2} \\
& \frac{m_{\tilde{\tilde{\varepsilon}}}^{2}}{m_{\tilde{g}}^{2}}=0.0008+0.104\left(\frac{m}{\eta m_{0}}\right)^{2}
\end{aligned}
$$

For $\eta \mathrm{m}_{0}=\mathrm{m}$ we obtain $\mathrm{m}_{\mathbf{q}} \simeq 0.99 \mathrm{~m}_{\mathrm{g}}$ and $\mathrm{w}_{\tilde{l}_{\mathrm{R}}} \simeq 0.32 \mathrm{w}_{\mathbf{g}}$. Using the PETRA/PEP bound $\mathrm{m}_{\mathrm{e}_{\mathrm{R}}}>18 \mathrm{GeV}$ we find that

$$
\begin{aligned}
& m_{\tilde{g}} \geqslant 55.6 \mathrm{GeV} \\
& m_{\tilde{q}} \geqslant 55.2 \mathrm{GeV}
\end{aligned}
$$

Therefore, the simplest possibility favours a two-squark or -gluino explanation $^{12)}$ of the UAl monojet events. Another explanation ${ }^{13)}$ of the monojet events has a light $[\sim 0(5) \mathrm{GeV}]$ gluino and $0(100) \mathrm{GeV}$ squarks. This spectrum can be realized if $\mathrm{m} / \eta \mathrm{m}_{0} \simeq 62$ : a possibility we cannot exclude although it appears surprising.

\section{ACKNOWLEDGEMENTS}

One of us (K.E.) gratefully acknowledges the financlal support of the Academy of Finland. Another (K.T.) wishes to express his gratitude to the MaxPlanck-Institut-Munich, and especially to Profs. W. Zimmerman and A. Buras for thefr hospitality and financial support. 


\section{REFERENCES}

1) G. Arnison et al. (UAl Collaboration), Phys. Lett. 122B (1983) 103 and 126B (1983) 398;

M. Banner et a1. (UA2 Collaboration), Phys. Lett. 122B (1983) 473;

P. Bagnaia et a1. (UA2 Collaboration), Phys. Lett. 129B (1983) 130.

2) For reviews, see

J. Ellis, CERN preprint TH.3802 (1984);

D.V. Nanopoulos, CERN preprint TH.3995 (1984);

H.P. Nilles, Phys. Rep. C110 (1984) 2.

3) J. Ellis, A.B. Lahanas, D.V. Nanopoulos and K. Tamvakis, Phys. Lett. 134B (1984) 429;

J. Ell1s, C. Kounnas and D.V. Nanopoulos, Nucl. Phys. B241 (1984) 406 and Nucl. Phys. B247 (1984) 373; Phys. Lett. 143B (1984) 410;

J. Ellis, K. Enqvist and D.V. Nanopoulos, Phys. Lett. 147B (1984) 99; ibid. 151B (1985) 357.

4) E. Cremmer, B. Julia, J. Scherk, S. Ferrara, L. Girardello and P. van Nieuwenhulzen, Phys. Lett. 79B (1978) 231; Nucl. Phys. B147 (1979) 105 ;

E. Cremmer, S. Ferrara, L. Girarde11o and A. Van Proeyen, Phys. Lett. 116B (1982) 231; Nucl. Phys. B212 (1983) 413.

5) B. Grinstein, Nucl. Phys B206 (1982) 387;

A. Masiero, D.V. Nanopoulos, K. Tamvak1s and T. Yanagida, Phys. Lett. 115B (1982) 380 .

6) J. E1lis, C. Kounnas and D.V. Nanopoulos, Nuc1. Phys. B247 (1984) 373.

7) J. E11is and S. Rudaz, Phys. Lett. 128B (1983) 248.

8) See e.g., K. Winter, CERN preprint EP/84-137 (1984).

9) D.V. Nanopoulos and D.A. Ross, Phys. Lett. 118B (1982) 99.

10) C. Kounnas, A.B. Lahanas, D.V. Nanopoulos and M. Quirós, Phys. Lett. 132B (1983) 95; Nuc1. Phys. B236 (1984) 438.

11) J. E11is and M. Sher, Phys. Lett. 148B (1984) 309;

L.E. Ibañez, C. Lopez and C. Muñoz, CERN preprint TH.4071 (1984);

K. Enquist, A.B. Lahanas and D.V. Nanopoulos, CERN preprint TH.4095 (1984).

12) J. Ellis and H. Kowalskf, Phys. Lett. 142B (1984) 441; Nucl. Phys. B246 (1984) 189; CERN preprint TH.4072 (1984);

E. Reya and D.P. Roy, Phys. Lett. 141B (1984) 42; Phys. Rev. Lett. 52 (1984) 881 .

13) M.J. Herrero et a1., Phys. Lett. 132B (1983) 199 and 145B (1984) 430;

v. Barger et al., Phys. Rev. Lett. 53 (1984) 641 and Univ. of Wisconsin preprint MAD/PH/197 (1984);

A. De Rújula and R. Petronz1o, CERN preprint TH.4070/84 (1984). 


\section{FIGURE CAPTIONS}

Fig. 1: The unification mass $M_{X}$ and $\sin ^{2} \theta_{W}$ as functions of $\eta / \xi[$ see (29)]. Shown also is the experimentally allowed range of $\sin ^{2} \theta_{W}$.

Fig. 2: The combination $\eta \alpha_{G U T}=5 \alpha_{3}\left(\mathbb{M}_{X}\right)$ as a function of $\eta / \xi[$ see (29)]. 


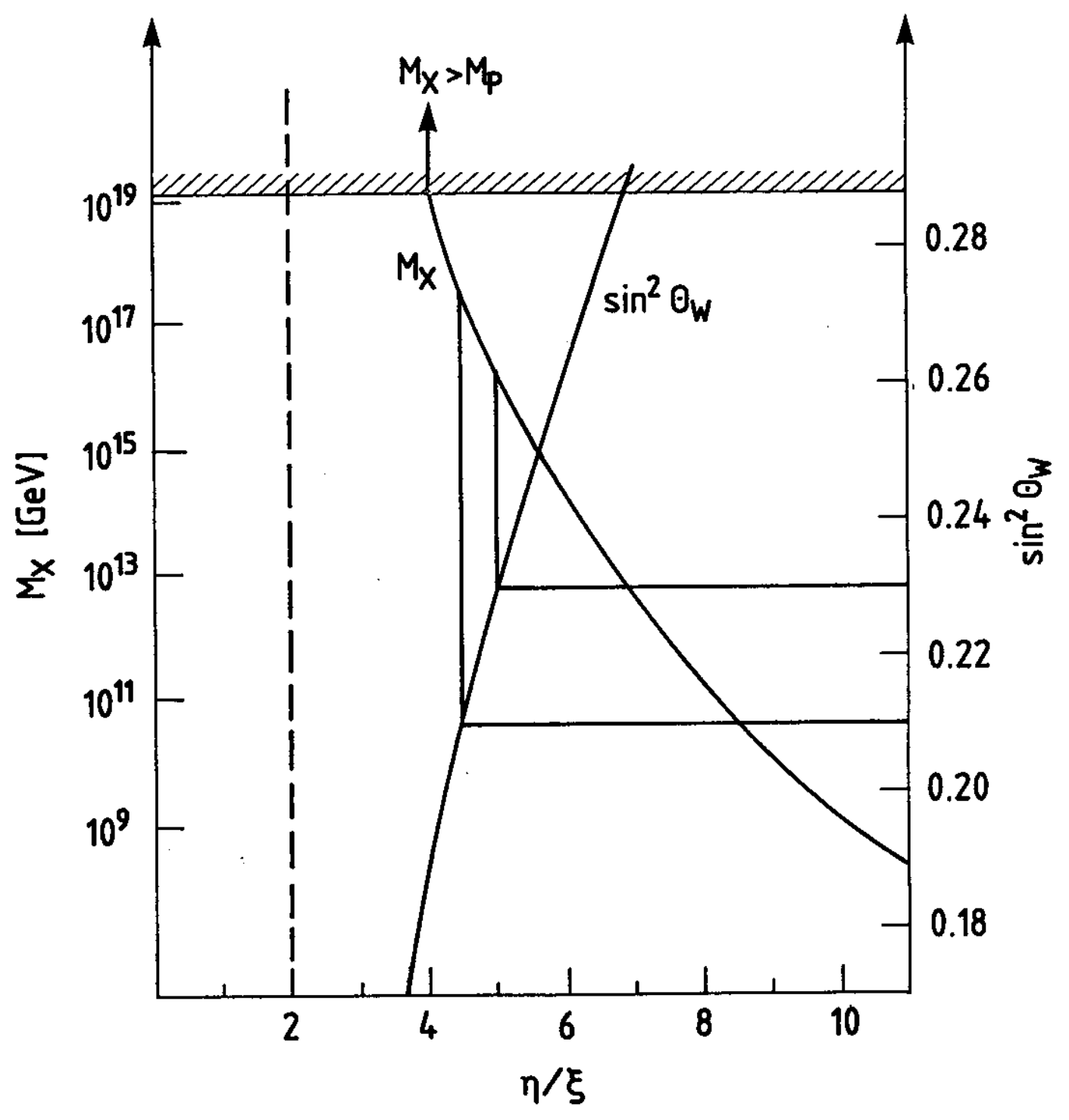

Fig. 1 


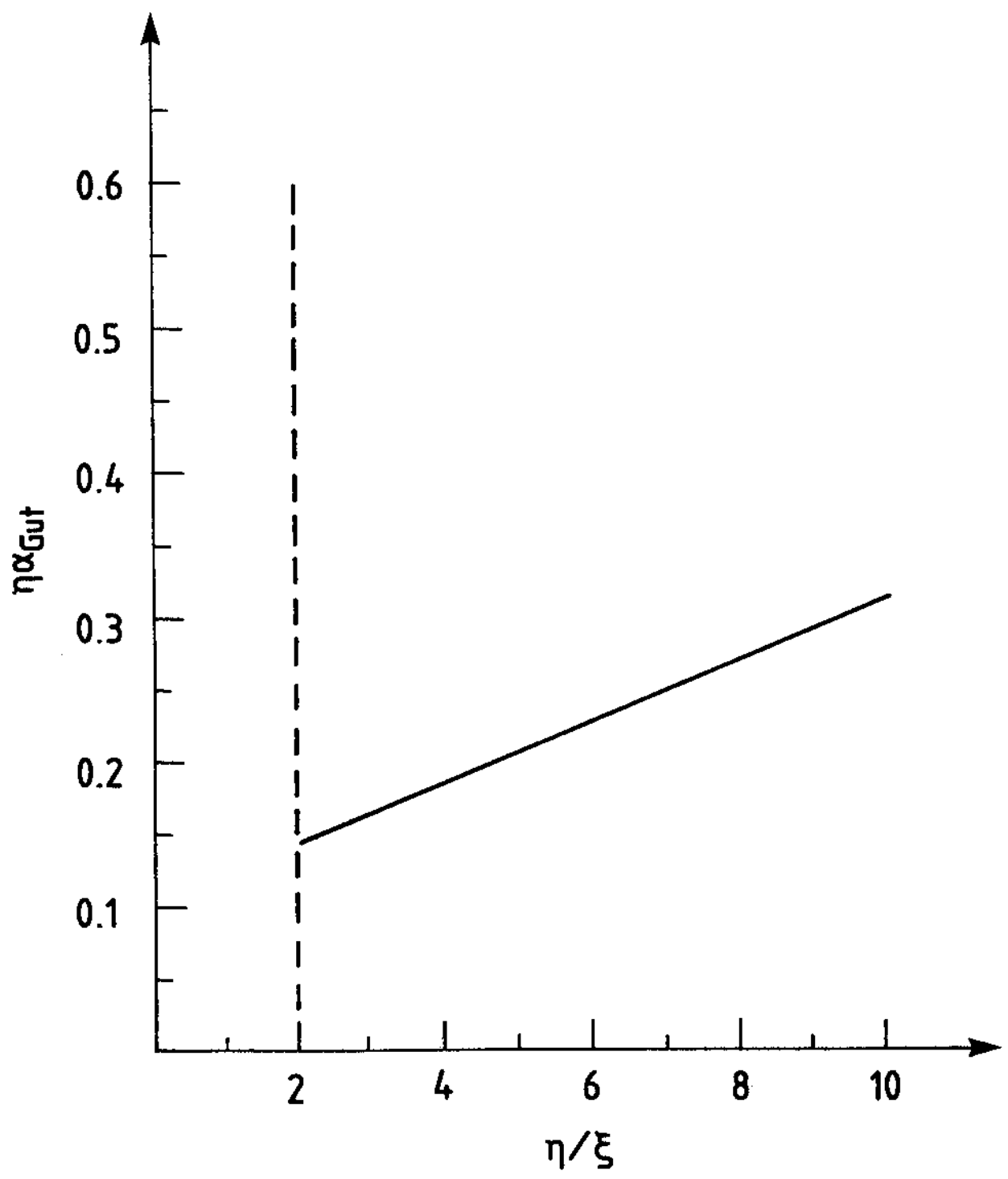

Fig. 2 